\title{
Methodology of Resonant Equiangular Composite Quantum Gates
}

\author{
Guang Hao Low, Theodore J. Yoder, and Isaac L. Chuang \\ Department of Physics and Research Laboratory of Electronics, Massachusetts Institute of Technology, \\ Cambridge, Massachusetts 02139, USA
}

(Received 12 March 2016; revised manuscript received 27 October 2016; published 28 December 2016)

The creation of composite quantum gates that implement quantum response functions $\hat{U}(\theta)$ dependent on some parameter of interest $\theta$ is often more of an art than a science. Through inspired design, a sequence of $L$ primitive gates also depending on $\theta$ can engineer a highly nontrivial $\hat{U}(\theta)$ that enables myriad precision metrology, spectroscopy, and control techniques. However, discovering new, useful examples of $\hat{U}(\theta)$ requires great intuition to perceive the possibilities, and often brute force to find optimal implementations. We present a systematic and efficient methodology for composite gate design of arbitrary length, where phase-controlled primitive gates all rotating by $\theta$ act on a single spin. We fully characterize the realizable family of $\hat{U}(\theta)$, provide an efficient algorithm that decomposes a choice of $\hat{U}(\theta)$ into its shortest sequence of gates, and show how to efficiently choose an achievable $\hat{U}(\theta)$ that, for fixed $L$, is an optimal approximation to objective functions on its quadratures. A strong connection is forged with classical discrete-time signal processing, allowing us to swiftly construct, as examples, compensated gates with optimal bandwidth that implement arbitrary single-spin rotations with subwavelength spatial selectivity.

DOI: 10.1103/PhysRevX.6.041067

\section{INTRODUCTION}

Composite quantum gates [1,2] are indispensable to many important quantum technologies, such as nuclear magnetic resonance, magnetic resonance imaging, quantum sensing, and quantum computation [3]. Their versatility arises from cunningly chosen sequences of $L$ primitive quantum gates that produce an effective quantum gate $\hat{U}$ with a more desirable dependence on some parameters of interest $\theta$, such as drive amplitude or background magnetic fields. As a function of $\theta$, the quantum response function $\hat{U}(\theta)$ can be tailored to amplify weak signals beyond the statistics of repetition and suppress noise without measurement. Finding such useful composite gates is thus the subject of intense research.

Even in single-spin systems, the focus of this work, extraordinary richness can be found in the possible forms of $\hat{U}(\theta)$. Examples include the following: NMR spectroscopy, where minute chemical shifts $\theta$ are made clearer through $\hat{U}(\theta)[2,4,5]$; Heisenberg-limited quantum imaging, where $\hat{U}(\theta)$ is made sensitive to subwavelength position variations $\theta[6]$ without aliasing [7,8]; subwavelength spatial addressing, where arbitrary quantum gates $\hat{U}(\theta)$ with low crosstalk are applied on spin arrays [8-10]; quantum phase estimation for atomic clocks [11] or tomography [12],

Published by the American Physical Society under the terms of the Creative Commons Attribution 3.0 License. Further distribution of this work must maintain attribution to the author(s) and the published article's title, journal citation, and DOI.
Subject Areas: Quantum Information

where extremely small drifts $\theta$ are amplified by a factor $L$ in the gradient of $\hat{U}(\theta)$; error compensation, where fractional control errors $\theta$ are exponentially suppressed like $\hat{U}(\theta)=$ $\hat{U}(0)+\mathcal{O}\left(\theta^{\text {poly }(L)}\right)$ [13-20]; and quantum algorithms such as amplitude amplification [21,22], where a computation $\hat{U}(\theta)$ proceeds with input $\theta$. The discovery of other applications would be expedited if a useful characterization of all achievable $\hat{U}(\theta)$ could be found.

However, the road to new results does not end with a choice of $\hat{U}(\theta)$. Given some reasonable system-dependent quantum control [23], its realization as a composite gate must be found. Only with rare exceptions $[8,9,16]$ and great effort are optimal arbitrary-length examples found in closed form. Thus, celebrated techniques including gradient ascent algorithms [5] and pseudospectral methods $[24,25]$ formulate this as a systematic optimization problem that can be solved by brute force but unfortunately with an exponential worst-case runtime $\mathcal{O}\left(e^{L}\right)$ for finding optimally short $L$ approximations. Finding efficient solutions to various control problems would expand the potential of long composite gates, for which the most sophisticated quantum response functions can be constructed.

A tantalizing similarity is seen in discrete-time signal processing [26]. Optimal finite-impulse response filters [27] can be designed simply by choosing the lowest-degree $L$ polynomial that is the optimal approximation to a desired frequency response, from which an optimal and exact implementation is computed-made possible by efficient algorithms for both steps. It is recognized that composite gates implement a filter on physical parameters $[20,28]$, 
and the use of polynomials in quantum response functions is well known [29,30]. Unfortunately, quantum constraints can render computing these polynomials and their optimal implementation a hard problem. It would be a tremendous advance if efficient solutions to these problems could be found and even more so if the countless results from the exalted history of classical discrete-time signal processing were transferable to the quantum realm.

One noteworthy step in this direction is the Shinnar-Le Roux algorithm [31,32] and its refinements [33-35], which have so far been restricted to the field of magnetic resonance imaging. In this case, $\theta$ represents the amplitude of background magnetic fields and manifests as an offresonant rotation. Given otherwise perfect and arbitrary single-spin control, this approach enables the efficient design of $\hat{U}(\theta)$ by a connection to finite-impulse response filters. Unfortunately, extending the concept to situations with different controls and additional restrictions, particularly the case of on-resonant compensating pulse sequences, appears to have been difficult.

We contribute to this aspiration with a methodology, similar to the Shinnar-Le Roux algorithm, for designing composite gates built from $L$ resonant primitive gates acting on a single spin, all rotating by an angle $\theta$ but not necessarily with the same phase. We rigorously characterize the quantum response functions $\hat{U}(\theta)$ achievable in this manner, proving how we can efficiently choose the form with trigonometric polynomials of degree $L$ and how their optimal implementation with the shortest sequence of primitive gates can be efficiently computed. In the process, a connection is made with discrete-time signal processing that allows us to inherit some of its machinery. These powerful tools expedite composite gate design, which we demonstrate with three optimal important examples: (1) narrow-band and broadband composite population inversion gates, (2) compensated NOT gates with optimal bandwidth, and (3) spatially selective arbitrary single-spin rotations below the diffraction limit.

In Sec. II, we elucidate the controls available to our single-qubit system, and in Sec. III, we demonstrate how equiangular composite gates motivate the intuitive concept of choosing polynomials to explicitly define the quantum response function $\hat{U}(\theta)$. This is made rigorous and tractable in Sec. III A by a simple characterization of the space of achievable $\hat{U}(\theta)$ and, in Sec. III B, by showing how an optimal implementation of any such $\hat{U}(\theta)$ can be efficiently computed. We then show in Sec. III C how an achievable $\hat{U}(\theta)$ can be efficiently computed from a partial specification with polynomials that describe only the composite gate fidelity or transition probability response functions. In Sec. III D, this enables the efficient design of achievable $\hat{U}(\theta)$ by inheriting existing polynomials and efficient polynomial design algorithms from discrete-time signal processing. Together, these provide the methodology outlined in Sec. III E for the systematic and efficient design of composite quantum gates. Use of this methodology is demonstrated in Sec. IV with the creation of optimalbandwidth-compensated gates in Sec. IV B that provide an optimal solution in Sec. IV C to the problem of implementing subwavelength spatially selective arbitrary quantum gates. Further directions are discussed in Sec. V.

\section{MODEL}

The unitary quantum response function $\hat{U}(\theta)$ describes how a quantum system evolves under the influence of some parameter $\theta$ of interest. We consider the generic system of a resonantly driven single spin and present a construction for composite quantum gates that motivates a powerful approach for designing their implemented response functions.

A two-level system driven by time-dependent Rabi frequency $\Omega(t)$ and phase $\phi(t)$ is controlled by the Hamiltonian $\hat{H}_{c}(t)=(\Omega(t) / 2) \hat{\sigma}_{\phi(t)}$, where $\hat{\sigma}_{\phi}=$ $\cos (\phi) \hat{\sigma}_{x}+\sin (\phi) \hat{\sigma}_{y}$ and $\sigma_{x, y, z}$ are Pauli matrices. Taking $\phi(t)=\phi$ to be constant over time $\tau$, we generate the primitive rotation

$$
\hat{R}_{\phi}(\theta)=e^{-i(\theta / 2) \hat{\sigma}_{\phi}}, \quad \theta=\int_{0}^{\tau} \Omega(t) d t .
$$

Note that $\Omega(t)$ might only be partially under our control; thus, it contains an uncontrollable residual signal. Our parameter of interest is thus $\theta$, which captures the effects of both control and signal.

An equiangular composite gate of length $L$ is built from these primitive rotations, each with the same rotation amplitude $\theta$ but with varying phases $\vec{\phi}=\left(\phi_{1}, \ldots, \phi_{L}\right)$. This produces a $\theta$-dependent unitary $\hat{U}(\theta)$, or quantum response function, of the form

$$
\begin{aligned}
\hat{U}(\theta) & =\hat{R}_{\phi_{L}}(\theta) \hat{R}_{\phi_{L-1}}(\theta) \ldots \hat{R}_{\phi_{1}}(\theta), \\
& =\sum_{j=0}^{L}(-i)^{j} \sin ^{j}\left(\frac{\theta}{2}\right) \cos ^{L-j}\left(\frac{\theta}{2}\right) \hat{\Phi}_{L, j},
\end{aligned}
$$

where $\hat{\Phi}_{L, j}=\hat{\sigma}_{x}^{j}\left(\operatorname{Re}\left[\Phi_{L, j}\right] \hat{\mathbb{1}}+i \operatorname{Im}\left[\Phi_{L, j}\right] \hat{\sigma}_{z}\right)$, and the phase sums $\Phi_{L, j}$ are defined through the recurrence [19]

$$
\begin{aligned}
& \Phi_{k, j}=\Phi_{k-1, j}+\Phi_{k-1, j-1} e^{i(-1)^{j+1} \phi_{j},} \\
& \Phi_{0,0}=1, \quad \Phi_{0, j \neq 0}=0,
\end{aligned}
$$

performed over $j=0,1, \ldots, k$ and then $k=1,2, \ldots, L$.

Now, we make the crucial observation that $\hat{U}(\theta)$ is a polynomial of degree $L$ in $x \equiv \cos (\theta / 2)$ and $y \equiv \sin (\theta / 2)$, with a particularly elegant representation. Using the trigonometric relation $x^{2}+y^{2}=1, \hat{U}(\theta)$ has the form 


$$
\begin{aligned}
& \hat{U}(\theta) \\
& = \begin{cases}A(x) \hat{\mathbb{1}}+i B(x) \hat{\sigma}_{z}+i C(y) \hat{\sigma}_{x}+i D(y) \hat{\sigma}_{y} & L \text { odd } \\
A(x) \hat{\mathbb{1}}+i B(x) \hat{\sigma}_{z}+i x C(y) \hat{\sigma}_{x}+i x D(y) \hat{\sigma}_{y} & L \text { even }\end{cases}
\end{aligned}
$$

where $A(x), B(x), C(y)$, and $D(y)$ are polynomials of, at most, degree $L$ with coefficients $a_{k}, b_{k}, c_{k}$, and $d_{k}$ $(k=0,1, \ldots, L)$, respectively. In the following, $A, B, C$, and $D$ without arguments are understood to be functions of the $x, y$ seen in Eq. (4). As the tuple $(A, B, C, D)$ is an equivalent representation of $\hat{U}(\theta)$, we refer to both interchangeably. In particular, achievable tuples are those that can be realized by some composite gate of Eq. (2).

Definition 1.-(Achievable polynomial tuples). A tuple of polynomials $(A, B, C, D)$ is achievable if $\exists L \in \mathbb{N}$ and $\vec{\phi} \in \mathbb{R}^{L}$ s.t. $\hat{U}(\theta)=\hat{R}_{\phi_{L}}(\theta) \hat{R}_{\phi_{L-1}}(\theta) \ldots \hat{R}_{\phi_{1}}(\theta)$ has the form of Eq. (4).

We are often interested in only a few components of $(A, B, C, D)$. For example, the partial tuple $(A, \cdot, C, \cdot)$ fully defines the gate fidelity response function $F_{\chi}(\theta)=$ $\frac{1}{4}\left|\operatorname{Tr}\left[\hat{R}_{0}^{\dagger}(\chi) \hat{U}\right]\right|^{2}$ with respect to some target gate $\hat{R}_{0}(\chi)$.

$$
F_{\chi}(\theta)= \begin{cases}\left|\cos \left(\frac{\chi}{2}\right) A-\sin \left(\frac{\chi}{2}\right) C\right|^{2} & L \text { odd } \\ \left|\cos \left(\frac{\chi}{2}\right) A-x \sin \left(\frac{\chi}{2}\right) C\right|^{2} & L \text { even. }\end{cases}
$$

Similarly, $(A, B, \cdot, \cdot)$ or $(\cdot, \cdot, C, D)$ fully defines the transition probability response function $p(\theta)=|\langle 0|\hat{U}| 1\rangle|^{2}$,

$$
p(\theta)=1-A^{2}-B^{2}=\left(C^{2}+D^{2}\right) \begin{cases}1 & L \text { odd } \\ x^{2} & L \text { even. }\end{cases}
$$

We refer to a tuple with $n$ empty slots as an $n$-partial tuple. An $n$-partial tuple is achievable if it is consistent with some achievable tuple.

A brute-force approach to composite gate design is minimizing an objective function for $\hat{U}(\theta)$ over a space $L \in \mathbb{N}, \vec{\phi} \in \mathbb{R}^{L}$. Though useful examples have been discovered in this manner, such an approach is highly unappealing. In addition to being inefficient with a runtime $\mathcal{O}\left(e^{L}\right)$, there is no guarantee that a globally optimal solution will be found. Furthermore, the procedure provides little of the necessary insight into possible $\hat{U}(\theta)$ for envisioning further novel applications.

\section{SYSTEMATIC AND EFFICIENT DESIGN OF OPTIMAL COMPOSITE GATES}

The functional form of $\hat{U}(\theta)$ hints at a powerful methodology for composite gate design via choices of the polynomials $(A, B, C, D)$ of degree $L$. This ambition must solve long-standing problems:

(P1) An insightful characterization of achievable $(A, B, C, D)$ to eliminate the traditional guesswork in envisioning novel quantum response functions and their dependence on $\vec{\phi}$.

(P2) An efficient algorithm to compute the optimal $\vec{\phi}$ implementing an achievable $(A, B, C, D)$, in contrast to the intractable random search in time $\mathcal{O}\left(e^{L}\right)$ of current state of the art [8].

(P3) An efficient algorithm to compute an achievable $(A, B, C, D)$ from achievable partial tuples, e.g., $(A, \cdot C, \cdot)$, as might be encountered with common objective functions for Eqs. (5) and (6).

(P4) An efficient algorithm for computing achievable partial tuples optimal for some objective function.

Our main technical advances are precisely the resolution of problems (1-4). We describe, in a simple and intuitive manner, the set of achievable $(A, B, C, D)$ and provide efficient algorithms for solving what have traditionally been the hardest aspects of composite gate design. In particular, a beautiful connection is made with the historic field of discrete-time signal processing that allows us to inherit much of its prior work in polynomial design. In this manner, the inspired art of composite gates is transformed into a systematic science. Optimal composite gates are simply polynomials that are optimal for the objective function, and these polynomials can be found efficiently.

\section{A. Characterization of quantum response functions}

Here, we characterize achievable choices of quantum response functions $(A, B, C, D)$ in a manner independent of $\vec{\phi}$, hence resolving problem (P1). By providing insight into the forms of possible $\hat{U}(\theta)$, we also obtain a quantitative explanation for the remarkable versatility of composite gates. Achievability constraints on the polynomials $(A, B, C, D)$ are as follows:

Theorem 1 (Achievable tuples). A tuple of polynomials $(A, B, C, D)$ of, at most, degree $L$ is achievable iff all the following are true:

(1) $A, B, C, D$ are real.

(2) $A(1)=1$ or $B(1)=0$.

(3) $\begin{cases}A, B, C, D \text { are odd } & L \text { odd, } \\ A, B \text { are even and } C, D \text { are odd } & L \text { even. }\end{cases}$

(4) $1= \begin{cases}A(x)^{2}+B(x)^{2}+C(y)^{2}+D(y)^{2} & L \text { odd } \\ A(x)^{2}+B(x)^{2}+x^{2} C(y)^{2}+x^{2} D(y)^{2} & L \text { even. }\end{cases}$

Proof.-In the forward direction, (1) and (3) are true if we apply the trigonometric substitution $x^{2}+y^{2}=1$ in Eq. (2) and collect coefficients of $\hat{\mathbb{1}}, \hat{\sigma}_{x, y, z}$. Case (2) is true as $\hat{U}(0)=\hat{\mathbb{1}}$ in Eq. (2). Case (4) is true as $\hat{U}$ is unitary, so $\hat{U}^{\dagger} \hat{U}=\hat{\mathbb{1}}$ and $\frac{1}{2} \operatorname{Tr}\left[\hat{U}^{\dagger} \hat{U}\right]$ evaluated via Eq. (4) produces

$$
1= \begin{cases}A(x)^{2}+B(x)^{2}+C(y)^{2}+D(y)^{2} & L \text { odd } \\ A(x)^{2}+B(x)^{2}+x^{2} C(y)^{2}+x^{2} D(y)^{2} & \text { Leven. }\end{cases}
$$


In the reverse direction, we need to show that any $(A, B, C, D)$ satisfying (1-4) is achievable in the sense of Definition 1. We leave these steps to Lemma 1.

Conditions (1-4) for achievable $(A, B, C, D)$ appear fairly general, which allows for great flexibility in choosing arbitrary response functions. They are also understandable and intuitive. A characterization of achievable partial tuples is also useful. Not all quadratures of $\hat{U}(\theta)$ might be relevant to an objective function, and optimizing over a subset $(A, B, C, D)$ could be easier. In the following, we examine how the unitarity constraint of condition (4) is weakened for all possible 2-partial tuples.

Theorem 2 (Achievable 2-partial tuples). Assuming A, $B, C, D$ satisfy conditions (1-3) of Theorem 1 ,

(1) $(A, \cdot, C, \cdot),(A, \cdot, \cdot, C)$ is achievable iff
(i) $\forall \theta \in \mathbb{R},\left\{\begin{array}{l}A(x)^{2}+C(y)^{2} \leq 1 \\ A(x)^{2}+x^{2} C(y)^{2} \leq 1\end{array}\right.$
$L$ odd

(2) $(\cdot, B, C, \cdot),(\cdot, B, \cdot, C)$ is achievable iff
(i) $\forall \theta \in \mathbb{R},\left\{\begin{array}{l}B(x)^{2}+C(y)^{2} \leq 1 \\ B(x)^{2}+x^{2} C(y)^{2} \leq 1\end{array}\right.$
$L$ odd

(3) $(A, B, \cdot, \cdot)$ is achievable iff

(i) $\forall \theta \in \mathbb{R}, A(x)^{2}+B(x)^{2} \leq 1$, and

(ii) $\forall x \geq 1, A(x)^{2}+B(x)^{2} \geq 1$, and

(iii) $\forall L$ even, $\quad \forall x \geq 0, A(i x)^{2}+B(i x)^{2} \geq 1$.

(4) $(\cdot, \cdot, C, D)$ is achievable iff

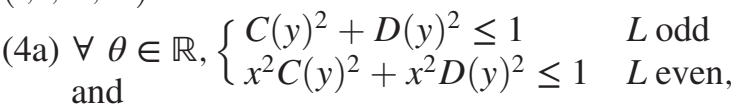
(4b) $\forall L$ odd, $y \geq 1, C(y)^{2}+D(y)^{2} \geq 1$.

Proof.-In the forward direction, all the (a) conditions are true from Eq. (7) using the fact that $A, B, C, D$ are all real; hence, their squares are positive. Condition (3b) is true if we consider Eq. (7) with the substitution $x=\sqrt{\lambda}$, $y=\sqrt{1-\lambda}$, and compute $1-A^{2}(\sqrt{\lambda})-B^{2}(\sqrt{\lambda})=\cdots$. Note that the $x, y$ here are complex. Using the odd/even symmetry of $C, D$, the rhs factorizes into a positive term times $(1-\lambda)$ or $\lambda(1-\lambda)$. This is negative $\forall \lambda \geq 1$ so $A^{2}(\sqrt{\lambda})+B^{2}(\sqrt{\lambda}) \geq 1$. Condition (3c) is similarly proven by considering $\lambda \leq 0$. The rhs factorizes into $\lambda(1-\lambda)$ and a positive term. Condition (4b) is proven with the substitution $x=\sqrt{1-\lambda}, \quad y=\sqrt{\lambda}$ and by considering $\lambda \geq 1$. In the reverse direction, we need to show that assuming these conditions enables the computation of an achievable $(A, B, C, D)$. We leave these steps to Lemmas 2 and 3.

Note that $C, D$ are interchangeable in Theorem 2 as their constraints in Theorem 1 are identical. We also characterize all possible 3-partial tuples.

Theorem 3 (Achievable 3-partial tuples). Assuming $A, B, C, D$ satisfy conditions (1-3) of Theorem 1, the following are achievable under their respective conditions:

(1) $(A, \cdot, \cdot, \cdot)$ iff $\forall \theta \in \mathbb{R}, A(x)^{2} \leq 1$.

(2) $(\cdot, B, \cdot, \cdot)$ iff $\forall \theta \in \mathbb{R}, B(x)^{2} \leq 1$.

(3) $(\cdot, \cdot, C, \cdot)$ iff $\forall \theta \in \mathbb{R}, \begin{cases}C^{2}(y) \leq 1 & L \text { odd } \\ x^{2} C^{2}(y) \leq 1 & L \text { even. }\end{cases}$
(4)
$\cdot, \cdot, \cdot, D)$ iff $\forall \theta \in \mathbb{R},\left\{\begin{array}{l}D^{2}(y) \leq 1 \\ x^{2} D^{2}(y) \leq 1\end{array}\right.$
$L$ odd
$L$ even

Proof.-The forward direction follows by definition and from Eq. (7), where $A, B, C, D$ are all real $\forall \theta \in \mathbb{R}$; hence, their squares are positive. The reverse direction is true from setting the unspecified polynomial to 0 in one of the 2-partial tuples (1) and (2) in Theorem 2.

These simple characterizations show how one can, in principle, encode almost any arbitrary desired function into quadratures of $\hat{U}(\theta)$. Consider $(A, \cdot, \cdot, \cdot)$, which aside from symmetry and $A(1)=1$, only needs to satisfy $\forall|x| \leq 1$, $A^{2}(x) \leq 1$. The famous Stone-Weierstrass theorem [36] assures us that $A(x)$ of sufficiently large degree $L$ can approximate arbitrarily well any arbitrary continuous real function that satisfies these constraints on the interval $|x| \leq 1$. This ability to create almost arbitrary quantum response functions helps explain the applicability of composite gates to many diverse problems.

\section{B. Implementation of quantum response functions}

Unleashing the potential of arbitrarily sophisticated choices of achievable $(A, B, C, D)$ requires an efficient computation of their implementation $\vec{\phi}$. It is clear that a random search is wholly inadequate, as the degree of $L$ could be very large. Nevertheless, achievability leads to a certain structure that resolves problem (P2). This is encapsulated in the following lemma, which is proven constructively and furnishes the reverse-direction proof of Theorem 1.

Lemma 1 (Optimal quantum response compilation). Exactly $L$ phases $\vec{\phi} \in \mathbb{R}^{L}$ are required to implement an achievable $(A, B, C, D)$ of, at most, degree $L$, and these $L$ phases can be computed in time $\mathcal{O}[\operatorname{poly}(L)]$.

Proof.-A minimum of $L$ phases $\vec{\phi}$ are required to implement a given $(A, B, C, D)$ of, at most, degree $L$, as each application of $\hat{R}_{\phi_{k}}(\theta)$ only increases the degree of $(A, B, C, D)$ by 1 . We now show that $(A, B, C, D)$ can be implemented with, at most, $L$ phases $\vec{\phi}$. Because of the even/odd symmetry of real $A, B, C, D$ from Theorem 1 , conditions (1) and (3), we can compute its unique phase sum representation in Eq. (2) via the invertible transformation

$$
\Phi_{L, j}=i^{j} \sum_{n=0}^{L} \begin{cases}\left(i c_{n}-d_{n}\right)\left(\begin{array}{l}
\lfloor(L-n) / 2\rfloor \\
(j-n) / 2
\end{array}\right) & j \text { odd } \\
\left(a_{n}+i b_{n}\right)\left(\begin{array}{c}
(L-n) / 2 \\
j / 2
\end{array}\right) & j \text { even }\end{cases}
$$

Let us take the ansatz $\hat{U}(\theta)=\hat{R}_{\phi_{L}}(\theta) \hat{V}(\theta)$, where $\hat{V}(\theta)$ is unitary and $\hat{V}(0)=\mathbb{1}$, as $(A, B, C, D)$ represents a unitary from Theorem 1, condition (4). Thus, $\hat{V}(\theta)$ also has a phase sum representation $\Phi_{L-1, j}$. These two phase sums are related by the linear map of Eq. (3), with inverse 


$$
\Phi_{L-1, j}=\sum_{k=0}^{j} \Phi_{L, k} \begin{cases}-e^{-(-1)^{j} i \phi_{L}} & j+k \text { odd } \\ 1 & j+k \text { even }\end{cases}
$$

By choosing

$$
e^{i \phi_{L}}=\frac{\sum_{k=1 \text { odd }}^{L} \Phi_{L, k}}{\sum_{k=0 \text { even }}^{L} \Phi_{L, k}}=\frac{c_{2\lceil L / 2\rceil-1}+i d_{2\lceil L / 2\rceil-1}}{(-1)^{\lceil L / 2\rceil}\left(a_{L}+i b_{L}\right)},
$$

we satisfy the necessary condition $\Phi_{L-1, L}=0$ from Eq. (3). In particular, $\phi_{L}$ is real, as Eq. (7) has the trailing term $\left[\left(a_{L}^{2}+b_{L}^{2}\right)-\left(c_{2\lceil L / 2\rceil-1}^{2}+d_{2\lceil L / 2\rceil-1}^{2}\right)\right] \sin ^{2 L}(\theta / 2)=0$. Hence, the rhs of Eq. (10) has absolute value 1. By recursively reducing the degree of $\hat{V}(\theta)$, we obtain all $L$ phases $\vec{\phi}$. The terminal case at $L=1$ must be consistent with Eq. (3), where $\Phi_{0,0}=1$. When evaluated with Eqs. (8) and (9), this is satisfied only if $A(1)=1$ [Theorem 1, condition (2)], which is true for achievable $(A, B, C, D)$. All steps in this procedure can be computed in time $\mathcal{O}[\operatorname{poly}(L)]$, and there are only $L$ recursions, leading to a runtime of $\mathcal{O}[\operatorname{poly}(L)]$.

\section{Computation of quantum response functions}

A consequence of Lemma 1 is that designing a composite gate is no more difficult than finding the $(A, B, C, D)$ to describe the quantum response function $\hat{U}(\theta)$. Optimizing $(A, B, C, D)$ for some objective function is far more intuitive than the prior art of a random search over $\vec{\phi}$. However, this still is a difficult problem. The unitary constraint Eq. (7) represents a system of quadratic multinomial equations that would have to be solved at each step of the optimization to obtain an achievable $(A, B, C, D)$. Solving such systems is, in general, a NPcomplete task. This is the essence of problem (P3): It would be much easier to optimize a subset of $(A, B, C, D)$, and doing so is often the problem of practical interest anyway.

This subset optimization is illustrated by the response functions $F_{\chi}(\theta), p(\theta)$ of Eqs. (5) and (6), which depend on only two polynomials. Optimizing just these for some objective function offers more freedom as the unitary constraint Eq. (7) is weakened to that of Theorem 2. Ultimately, we must compute some achievable $(A, B, C, D)$ from a partial specification in order to find the phases $\vec{\phi}$.

Fortunately, the structure of achievable partial tuples can be exploited to derive algorithms analogous to prior art [31] based on polynomial sum-of-squares problems [37] but specialized to the symmetries of Theorem 2 . We present results for $(A, B \cdot, \cdot),(A, \cdot, C, \cdot)$ of odd degree and show how they apply to all achievable 2-partial tuples. As these primarily serve to show that the necessary conditions in Theorems 1, 2, and 3 are also sufficient, the details of the proofs for Lemmas 2 and 3, which also furnish constructive algorithms for computing $(A, B, C, D)$ from partial tuples, may be skipped by the casual reader.

Lemma 2 (Transition probability sum of squares). $\forall 2$ partial tuples $(A, B, \cdot, \cdot)$ of, at most, odd degree $L$ that satisfy conditions (1-3) of Theorem 1 and (3a, 3b) of Theorem 2, $\exists$ achievable $(A, B, C, D)$ of, at most, degree $L$ that can be computed in time poly $(L)$.

Proof.-Consider the polynomial of, at most, degree $L$,

$f(\lambda)=1-A^{2}(\sqrt{1-\lambda})-B^{2}(\sqrt{1-\lambda}), \quad \lambda \in \mathbb{R}$,

with roots $S=\{s \mid f(s)=0\} \in \mathbb{C}^{L}(S$ contains duplicates if a root is degenerate). Since $A, B$ are odd polynomials, $f(\lambda)$ is real for all real $\lambda$. Because $f(\lambda)$ is real, complex roots $s, s^{*}$ occur in pairs. Thus, we can group subsets of $S$ without loss of generality as

$S_{0}=\{s \in S \mid s=0\}, \quad S_{\mathrm{c}}=\{s \in S \mid \operatorname{Im}[s]>0\}$,

$S_{\mathrm{r}}=\{s \in S \mid \operatorname{Re}[s] \neq 0 \wedge \operatorname{Im}[s]=0\}$.

Observe that $S_{0, r}$ are real, and $S_{c}$ is complex. Thus,

$f(\lambda)=K^{2} \lambda^{\left|S_{0}\right|} \prod_{s \in S_{\mathrm{r}}}(\lambda-s) \prod_{s \in S_{\mathrm{c}}}\left[(\lambda-\operatorname{Re}[s])^{2}+\operatorname{Im}[s]^{2}\right]$,

with scale constant $K \in \mathbb{R}$. Using (3b), $f(\lambda) \leq 0, \forall \lambda \leq 0$. Hence, all negative roots in $S_{\mathrm{r}}$ occur with even multiplicity. Using (3a), $f(\lambda) \in[0,1], \forall \lambda \in[0,1]$. As $f(\lambda)$ changes sign at $\lambda=0,\left|S_{0}\right|$ is odd. Using the oddness of $A, B$, $f(\lambda) \geq 1, \forall \lambda \geq 1$. Since $f(\lambda) \geq 0, \forall \lambda \geq 0$, all positive roots in $S_{\mathrm{r}}$ occur with even multiplicity. Thus, all real roots excluding $s=0$ occur with even multiplicity. By repeated application of the two-squares identity

$$
\left(r^{2}+s^{2}\right)\left(t^{2}+u^{2}\right)=(r t \pm s u)^{2}+(r u \mp s t)^{2},
$$

the complex factors can be simplified as

$$
\prod_{s \in S_{\mathrm{c}}}\left((\lambda-\operatorname{Re}[s])^{2}+\operatorname{Im}[s]^{2}\right)=g^{2}(\lambda)+h^{2}(\lambda),
$$

where $g, h$ are real polynomials in $\lambda$. Thus, $f(\lambda)=$ $C^{2}(\sqrt{\lambda})+D^{2}(\sqrt{\lambda})$, where

$$
\left\{\begin{array}{l}
C(y) \\
D(y)
\end{array}\right\}=\left(K y^{\left|S_{0}\right|} \prod_{s \in S_{\mathrm{r}}}\left(y^{2}-s\right)^{\frac{1}{2}}\right)\left\{\begin{array}{l}
g\left(y^{2}\right) \\
h\left(y^{2}\right)
\end{array}\right\},
$$

and $C, D$ are odd real polynomials of, at most, degree $L$. Note that the choice of signs in Eq. (14), generates a finite number of different valid solutions. Computing the roots of $f(\lambda)$ is the most difficult step of this algorithm, but it can be done in time $\mathcal{O}[\operatorname{poly}(L)]$ [38].

The proof for even $L$ and tuples $(\cdot, \cdot, C, D)$ carries through with minor modification. The stated conditions 
in Theorem 2 guarantee that the various factors of $\lambda,(1-\lambda)$ necessary for the correct symmetry of the unspecified polynomials occur with the right multiplicity and that all other real roots occur with even multiplicity. Some additional processing for the $(\cdot, \cdot, C, D)$ case is required, as the output $(A, B, C, D)$ is not guaranteed to satisfy $A(1)=1$. However, $A(1)^{2}+B(1)^{2}=1$ is still true, so by computing $\gamma=\arg [A(1)+i B(1)]$, we can form an achievable $(A \cos \gamma+B \sin \gamma, B \cos \gamma-A \sin \gamma, C, D)$.

We now present the analogous algorithm for $(A, \cdot, C, \cdot)$.

Lemma 3 (Fidelity response sum of squares). $\forall 2$ partial tuples $(A, \cdot, C, \cdot)$ of, at most, odd degree $L$ that satisfy conditions (1-3) of Theorem 1 and (1a) of Theorem 2, $\exists$ achievable $(A, B, C, D)$ of, at most, degree $L$ that can be computed in time poly $(L)$.

Proof.-With the Weierstrass substitution $\forall t \in \mathbb{R}$, $x=\left(1-t^{2}\right) /\left(1+t^{2}\right), y=2 t /\left(1+t^{2}\right)$ define the real polynomials

$$
(\tilde{A}(t), \tilde{B}(t), \tilde{C}(t), \tilde{D}(t))=\left(1+t^{2}\right)^{L}(A, B, C, D) .
$$

These polynomials have extremely useful symmetries, which we indicate with angled brackets $\langle\cdot\rangle$. Note that $\langle\tilde{A}\rangle=$ $\langle\tilde{B}\rangle=\langle E N\rangle$ are even $\langle\mathrm{E}\rangle$ antipalindromes $\langle\mathrm{N}\rangle$ while $\langle\tilde{C}\rangle=$ $\langle\tilde{D}\rangle=\langle O P\rangle$ are odd $\langle\mathrm{O}\rangle$ palindromes $\langle\mathrm{P}\rangle$. Antipalindromes satisfy $\tilde{A}(t)=-t^{2 L} \tilde{A}\left(t^{-1}\right)$, whereas palindromes satisfy $\tilde{C}(t)=t^{2 L} \tilde{C}\left(t^{-1}\right)$. Note that $\langle\mathrm{E}\rangle,\langle\mathrm{O}\rangle$ and $\langle\mathrm{P}\rangle,\langle\mathrm{N}\rangle$ polynomials with multiplication form a group isomorphic to $Z_{2} \times Z_{2}$. For example, $\langle\mathrm{EN}\rangle\langle\mathrm{OP}\rangle=\langle\mathrm{ON}\rangle$. Consider the positive, palindromic polynomial

$\tilde{f}(t)=\left(1+t^{2}\right)^{2 L}-\tilde{A}^{2}(t)-\tilde{C}^{2}(t)=K^{2} \prod_{s \in S}(t-s)$,

with scale constant $K \in \mathbb{R}$, and roots $S=\{s \mid \tilde{f}(s)=0\} \in$ $\mathbb{C}^{4 L-\left|S_{0}\right|}$, where $\left|S_{0}\right|$ is the multiplicity of the zero roots. Note that the degree of $\tilde{f}(t)$ is $4 L-\left|S_{0}\right|$, not $4 L$, because the first $\left|S_{0}\right|$ coefficients being zero implies the last $\left|S_{0}\right|$ are zero as well. Because of the $\langle E P\rangle$ symmetry of $\tilde{f}(t), \forall$ roots $s \neq 0, \exists$ roots $s^{*},-s$, and $s^{-1}$. Thus, we group subsets of these roots without any loss of information as follows:

$S_{0}=\{s \in S \mid s=0\}, \quad S_{1}=\{s \in S \mid s=1\}$,

$S_{r}=\{s \in S \mid \operatorname{Re}[s]>1 \wedge \operatorname{Im}[s]=0\}$,

$S_{i}=\{s \in S \mid \operatorname{Re}[s]=0 \wedge \operatorname{Im}[s]=1\}$,

$S_{l}=\{s \in S \mid \operatorname{Re}[s]=0 \wedge \operatorname{Im}[s]>1\}$,

$S_{u}=\{s \in S|| s \mid=1 \wedge 0<\arg [s]<\pi / 2\}$,

$S_{c}=\{s \in S|| s \mid>1 \wedge 0<\arg [s]<\pi / 2\}$.

Observe that $S_{0,1, r}$ are real, $S_{i, l}$ are imaginary, and $S_{u, c}$ are complex. From the real roots, we construct the factor

$$
\begin{aligned}
f_{\mathrm{r}} & =t^{\left(\left|S_{0}\right| / 2\right)}\left(t^{2}-1\right)^{\left(\left|S_{1}\right| / 2\right)} \prod_{s \in S_{r}}\left(t^{4}-t^{2}\left(s^{2}+s^{-2}\right)+1\right)^{\frac{1}{2}} \\
\left\langle f_{\mathrm{r}}\right\rangle & =\langle\mathrm{OP}\rangle^{\left(\left|S_{0}\right| / 2\right)}\langle\mathrm{EN}\rangle^{\left(\left|S_{1}\right| / 2\right)}\langle\mathrm{EP}\rangle^{\left(\left|S_{r}\right| / 2\right)} .
\end{aligned}
$$

The positiveness of $\tilde{f}(t)$ means that all real factors have even multiplicity. Thus, $f_{\mathrm{r}}$ is a polynomial. From the complex roots, we form

$$
\begin{aligned}
f_{\mathrm{i}}= & {\left[\left(t^{2}-1\right)^{2}+(2 t)^{2}\right]^{\left|S_{i}\right|}, } \\
f_{l}= & \prod_{s \in S_{l}}\left[\left(t^{2}-1\right)^{2}+\left(t\left(\operatorname{Im}[s]+\operatorname{Im}[s]^{-1}\right)\right)^{2}\right], \\
f_{\mathrm{u}}= & \prod_{s \in S_{\mathrm{u}}}\left[\left(t^{2}-1\right)^{2}+(2 t \sin (\arg [s]))^{2}\right], \\
f_{\mathrm{c}}= & \prod_{s \in S_{\mathrm{c}}}\left[\left(t^{4}-t^{2}\left(|s|^{-2}-4 \sin ^{2}(\arg [s])+|s|^{2}\right)+1\right)^{2}\right. \\
& \left.+\left(2\left(t^{3}+t\right) \operatorname{Im}[s]\left(1-|s|^{-2}\right)\right)^{2}\right] .
\end{aligned}
$$

The symmetry of terms under the squares is one of $\langle\mathrm{EP}\rangle$, $\langle\mathrm{EN}\rangle,\langle\mathrm{OP}\rangle,\langle\mathrm{ON}\rangle$, and it occur in a combination that forms a group under repeated application of the two-squares identity of Eq. (14). Thus, we can construct

$$
\begin{aligned}
f_{i} f_{l} f_{\mathrm{u}} f_{\mathrm{c}} & =g^{2}+h^{2}, \\
\langle g\rangle & =\langle\mathrm{EN}\rangle\left(\left|S_{i}\right| / 2\right)+\left|S_{\mathrm{u}}\right|+\left|S_{l}\right| \\
\langle h\rangle & =\langle\mathrm{OP}\rangle\left(\left|S_{i}\right| / 2\right)+\left|S_{\mathrm{u}}\right|+\left|S_{l}\right| \\
\tilde{f}(t) & =\left(K f_{\mathrm{r}} g\right)^{2}+\left(K f_{\mathrm{r}} h\right)^{2} .
\end{aligned}
$$

For some combinations of multiplicities, this decomposition will not produce polynomials with the symmetry $\langle\mathrm{EN}\rangle$, $\langle\mathrm{OP}\rangle$ required by $\tilde{B}, \tilde{D}$. However, summing the multiplicities of these roots shows that $\left|S_{i}\right|$ is even and that such combinations do not exist. From this decomposition, we compute $B(x), D(y)$ using

$$
\begin{aligned}
b_{k}= & \frac{1}{2^{L}} \sum_{n=0}^{L} \tilde{b}_{2 n}\left[\sum_{m=0}^{n}(-1)^{m}\left(\begin{array}{c}
n \\
m
\end{array}\right)\left(\begin{array}{c}
L-n \\
k-m
\end{array}\right)\right], \\
d_{2 k+1}= & \frac{(-1)^{k}}{2^{L}} \sum_{n=0}^{L-1} \tilde{d}_{2 n+1}\left[\sum_{m=0}^{n} \sum_{p=0}^{L / 2\rfloor}(-1)^{m}\left(\begin{array}{l}
p \\
k
\end{array}\right)\right. \\
& \left.\times\left(\begin{array}{c}
L-n-1 \\
2 p-m
\end{array}\right)\left(\begin{array}{c}
n \\
m
\end{array}\right)\right] .
\end{aligned}
$$

As with Lemma 2, different choices of signs in the twosquares identity lead to multiple valid solutions. Computing the roots of $\tilde{f}(t)$ is still the most difficult step, but it can be done in time $\mathcal{O}[\operatorname{poly}(L)]$.

The case of even $L$ replaces Eq. (18) with $\tilde{f}(t)=$ $\left(1+t^{2}\right)^{2 L}-\tilde{A}^{2}(t)-\left[\left(1-t^{2}\right) /\left(1+t^{2}\right)\right]^{2} \tilde{C}^{2}(t), \quad$ and we find $\tilde{B}$ with $\langle\mathrm{EP}\rangle$ and $\left(1-t^{2}\right) \tilde{D}$ with $\langle\mathrm{ON}\rangle$ symmetry. 
A similar root-counting argument guarantees the existence of such solutions. The coefficients of $B(x), D(y)$ are then computed using Eq. (23) also. This procedure carries through without modification for the other tuples (1), (2) of Theorem 2.

\section{Selection of quantum response functions}

It should be clear that optimal composite gate design is a systematic process no more difficult than choosing one or two polynomials that are optimal for some objective function. Nevertheless, problem (P4) is that computing these optimal polynomials could still be a difficult task. However, the constraints on achievable partial tuples in Theorems 2, 3 seem fairly lax, which lends hope that this could be done efficiently. In fact, these constraints are consistent with textbook problems in approximation theory [39].

It is at this point that a close connection with discretetime signal processing [26] is made. Efficient algorithms [40-44] for designing polynomials optimal for arbitrary objective functions under a variety of optimality criteria have been extensively studied for finite-impulse response filters [27]. We thus inherit much of this machinery, and in many cases, existing polynomials consistent with achievability have already been found and are directly transferable.

A most common optimality criterion is the Chebyshev norm: Let $P_{\mathrm{o}}(x)$ be the objective function, with continuous weight function $W(x)>0$, to be approximated by a polynomial $P(x)$ of degree $L$ on a bounded subset $\mathcal{B}$ of the closed interval $\mathcal{B} \subset[-1,1)$ with the smallest Chebyshev error norm

$$
\epsilon=\max _{x \in \mathcal{B}}\left|W(x)\left[P(x)-P_{\mathrm{o}}(x)\right]\right| .
$$

The unique best approximation can be computed efficiently by Remez-type exchange algorithms [45]. Many variants exist, such as where $P(x)$ is a trigonometric polynomial [40] that is bounded [42], subject to other unary or linear constraints [43], and even complex [41]. Linear programming methods [43] provide an alternate solution. Efficient algorithms for other optimality criteria such as least squares are also available [46,47].

These algorithms efficiently solve the problem of optimization over achievable quantum response functions $\hat{U}(\theta)$, where the objective functions are 2-partial or 3-partial tuples. Optimization for a 3-partial objective function involves a single quadrature from $(A, B, C, D)$, together with a single real objective function $P_{\mathrm{o}}(\theta)$. Thus, we optimize over $P(\theta)$ for $P_{\mathrm{o}}(\theta)$ in Eq. (24), subject to the constraints of Theorem 3 for the corresponding quadrature. The slightly more complicated 2-partial case instead specifies two quadratures and real objective functions $P_{\mathrm{o}, 1}(\theta), P_{\mathrm{o}, 2}(\theta)$. Thus, we define $P_{\mathrm{o}}(\theta)=P_{\mathrm{o}, 1}(\theta)+i P_{\mathrm{o}, 2}(\theta)$ and optimize over $P(\theta)=P_{1}(\theta)+i P_{2}(\theta)$ for $P_{\mathrm{o}}(\theta)$, subject to the constraints of Theorem 2 for the corresponding quadratures. Note that the unitarity inequality constraint poses no difficulty as $|P(\theta)|^{2}=P_{1}^{2}(\theta)+P_{2}^{2}(\theta)$.

\section{E. Methodology of composite quantum gates}

Our efforts lead us to a methodology for the design of single-spin quantum response functions $\hat{U}(\theta)$ through composite quantum gates built from a sequence of $L$ primitive gates all rotating by $\theta$, but each with its own phase $\vec{\phi}=\left(\phi_{1}, \ldots, \phi_{L}\right)$. The procedure is systematic, flexible, and most importantly, provably efficient:

Problem statement: Given $L \geq 1$ and objective function $\hat{U}_{o}(\theta)$ for either 3-partial or 2-partial tuples, find the composite quantum gate that implements through $\vec{\phi}$ the optimal $\epsilon$ approximation to $\hat{U}_{0}(\theta)$.

\section{Solution procedure:}

(S1) Check that $\hat{U}(\theta)$ is consistent with achievability.

Satisfies conditions of Theorems 2,3.

(S2) Choose an optimality criterion.

The Chebyshev norm is most common.

(S3) Execute a polynomial optimization algorithm over achievable partial tuples.

Remez-type algorithms are efficient.

(S4) Compute an achievable tuple from a partial tuple.

This can be done efficiently by Lemmas 2, 3 .

(S5) Compute phases $\vec{\phi}$.

This can be done efficiently by Lemma 1 .

\section{EXAMPLES}

Using the methodology in Sec. III E, composite quantum gates with the response function $\hat{U}(\theta)$ that minimize the error with respect to arbitrary objective functions $\hat{U}_{o}(\theta)$ can be efficiently designed. We illustrate this process with three examples of independent scientific interest: compensated population inversion gates, compensated broadband NOT gates, and compensated narrow-band quantum gates.

Population inversion gates rotate states $|0\rangle$ to $|1\rangle$ and vice versa, and come in two flavors. The broadband variant implements this rotation with high probability across the widest bandwidth of $\theta \in \mathcal{B}$, meaning that the transition probability response function $p(\theta)$ from Eq. (6) is close to 1 . The narrow-band variant instead implements this rotation with low probability, so $p(\theta) \approx 0$, except at a single point $p(\pi)=1$. We discuss the optimal design of these gates in Sec. IV A. As closed-form solutions for these gates are already known and used extensively in NMR spectroscopy, they help build familiarity with the methodology in Sec. III E when it is used to solve open questions in the next two examples.

Broadband compensated NOT gates implement the rotation $\hat{R}_{0}(\pi)$ with high fidelity over the widest bandwidth of $\theta$ parameters. Whereas population inversion gates only succeed on initial states $|0\rangle$ to $|1\rangle$, NOT gates apply a $\pi$ rotation with a known phase for all input states. Such gates 
have been extensively studied for applying uniform rotations in the presence of drive-field inhomogeneities, particularly in quantum computing applications, and our methodology, presented in Sec. IV B, solves open questions regarding the scaling of bandwidth with sequence length as well as their efficient synthesis.

A complementary design problem addressed in Sec. IV C is that of narrow-band compensated quantum gates. These instead apply a desired arbitrary rotation $\hat{R}_{0}(\chi)$ at a single $\theta$ value and the identity rotation elsewhere over the widest bandwidth of $\theta$ parameters. Such gates are highly relevant to minimizing crosstalk in the selective addressing of spins in arrays, particularly when spin-spin distances are below the diffraction limit, as might be found in scalable architectures of ion-trap quantum computation.

\section{A. Composite population inversion gates}

Population inversion gates maximize the bandwidth $\mathcal{B}$ over which the transition probability response function $p(\theta)$ from Eq. (6) is close to 1 for the broadband variant or close to 0 for the narrow-band variant. Note that in both cases, perfect population inversion occurs at $\theta=\pi$ for $L$ odd, owing to the fact that $A(0)=0$. Moreover, the optimal polynomials and phases for both variants turn out to be related by a simple transformation, so it suffices for us to consider only the broadband case.

Composite gates with these properties have been studied extensively for nuclear magnetic resonance and quantum computing applications. One approach to obtaining broadband behavior is with the maximally flat ansatz $p(\theta)=$ $1-\mathcal{O}\left[(\theta-\pi)^{2 n}\right]$ [9]. This exponentially suppresses errors in the transition probability to order $n$; thus, $p(\theta) \approx 1$ over a wide range of $\theta$. Remarkably, the $\vec{\phi}$ that implement this profile can be found in closed form [48] with optimal sequence lengths $L=n$. More recently, a second approach has emerged [8], motivated by the following observation: As the flat ansatz $p(\theta)=1-\mathcal{O}\left[(\theta-\pi)^{n}\right]$ only increases bandwidth indirectly through the suppression order $n$, better results can be obtained by directly optimizing for bandwidth while ensuring that the worst-case error $\mathcal{I}$ remains bounded.

The procedure of Sec. III E for odd $L$ formalizes this task as a straightforward optimization problem:

(S1) Choose the objective function $\forall \theta \in \mathcal{B}=$ $\pi+[-|\mathcal{B}| / 2,|\mathcal{B}| / 2], \hat{U}_{\mathrm{o}}(\theta)=0$ for the $(A, 0, \cdot, \cdot) 2-$ partial tuple. Since $p(\theta)=1-A^{2}$ is close to 1 over $\mathcal{B}$, the unitarity constraint $C^{2}+D^{2}=1-A^{2}$ implies that a rotation $R_{\phi}(\pi)$ is approximated over $\mathcal{B}$, with an unspecified phase $\phi=\arg [C+i D]$ that varies with $\theta$. As consistency with Theorem 1 requires that $A(1)=1$, this implies that identity is applied at $\theta=0$; thus, $\mathcal{B}$ must not contain $\theta=0$.

(S2) Choose the Chebyshev optimality criterion, where the best $A$ solves the minimax optimization problem

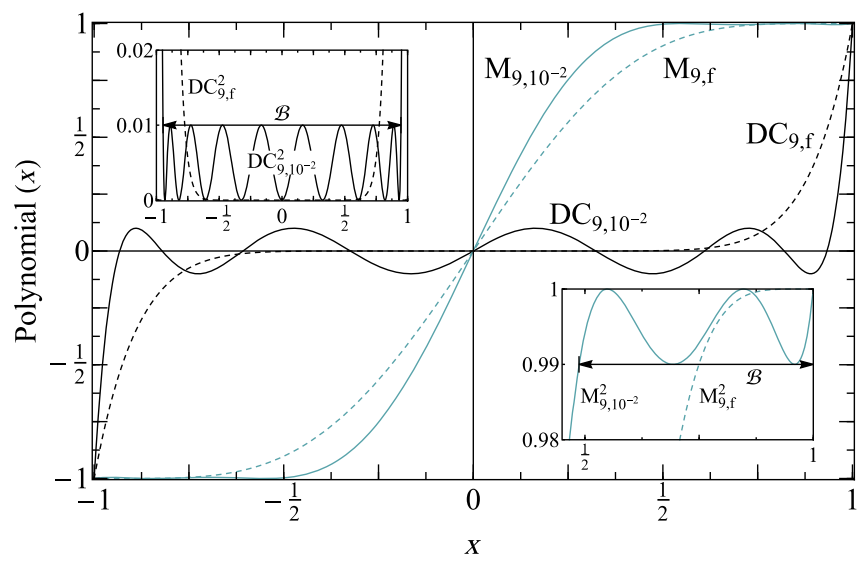

FIG. 1. $\mathrm{DC}_{L, \mathcal{I}}$ (black lines) and $\mathrm{M}_{L, \mathcal{I}}$ (teal lines) polynomials plotted for $L=9$ and target worst-case infidelity $\mathcal{I}=10^{-2}$ (solid lines) and $\mathcal{I} \rightarrow 0$ (dashed lines), indexed by $f$. The observed ripples are a generic feature of bandwidth-optimized polynomials, unlike those optimized for maximal flatness $\mathrm{DC}_{\mathrm{f}}, \mathrm{M}_{\mathrm{f}}$. The inset plots their squares and defines the bandwidth $\mathcal{B}$ in $x$ coordinates.

$$
\epsilon=\min _{A} \max _{\theta \in \mathcal{B}}|A(x)|, \quad \epsilon^{2}=\mathcal{I},
$$

where the worst-case transition probability over $\mathcal{B}$ is $1-\mathcal{I}$.

(S3) Find the function $A$ that solves Eq. (25). For consistency with Theorem 1, the optimization is over real odd polynomials $A$ bounded by $\forall|x| \lessgtr 1,|A(x)| \lessgtr 1$.

(S4) Using Lemma 2, compute the achievable tuple $(A, 0, C, D)$ from the partial specification $(A, 0, \cdot, \cdot)$. (S5) Compute $\vec{\phi}$ from $(A, 0, C, D)$ using Lemma 1.

The solution to (S3) is the Dolph-Chebyshev window function $[49,50]$ famous in discrete-time signal processing,

$\mathrm{DC}_{L, \mathcal{I}}(y)=\sqrt{\mathcal{I}} T_{L}\left(\beta_{L, \mathcal{I}} x\right), \quad \beta_{L, \mathcal{I}}=T_{L^{-1}}\left(\mathcal{I}^{-1 / 2}\right)$,

where $T_{n}(x)=\cos [n \arccos (x)]$ are Chebyshev polynomials. Note the ripples of $\mathrm{DC}_{L, \mathcal{I}}^{2}(x)$ bounded by $\mathcal{I}$ in Fig. 1. This is in contrast to a monotonic increase of the limiting function, indicated by the subscript $\mathrm{f}$,

$$
\mathrm{DC}_{L, \mathrm{f}}(x)=\lim _{\mathcal{I} \rightarrow 0} \mathrm{DC}_{L, \mathcal{I}}(x)=x^{L},
$$

which is maximally flat at $x=0$ but has a significantly narrower bandwidth. Using $x=\cos (\theta / 2)$, the bandwidth in $\theta$ coordinates is, to order $\mathcal{O}\left(\mathcal{I}^{(3 / 2 L)}\right)$,

$|\mathcal{B}|=2^{3-(1 / L)} \mathcal{I}^{(1 / 2 L)},\left|\mathcal{B}_{\mathrm{f}}\right|=4 \mathcal{I}_{\mathrm{f}}^{(1 / 2 L)}, \frac{\mathcal{I}}{\mathcal{I}_{\mathrm{f}}}=4^{1-L}$.

Given the same target bandwidth, the worst-case error of $\mathrm{DC}_{L, \mathcal{I}}$ is exponentially smaller than $\mathrm{DC}_{L, \mathrm{f}}$. Note also the quadratic difference in the scaling with $L$ of the bandwidth over which $\mathrm{DC}_{L, \mathcal{I}}$ does not approximate $F(x)=0$. 
$\overline{\mathcal{B}}=\frac{4 \operatorname{arcsech} \sqrt{\mathcal{I}}}{L}+\mathcal{O}\left(\frac{1}{L^{3}}\right), \quad \overline{\mathcal{B}}_{\mathrm{f}}=4 \sqrt{\frac{\log \frac{1}{\mathcal{I}}}{L}}+\mathcal{O}\left(\frac{1}{L^{\frac{3}{2}}}\right)$.

The ripples in the amplitude are a generic feature of the best polynomial approximations to functions in the Chebyshev norm. By sacrificing flatness, much smaller absolute variations in error $\epsilon$ can be achieved over some specified bandwidth $\mathcal{B}$. This is a common theme that will be revisited in the subsequent example. Finding the phases that implement $\left[\operatorname{DC}_{L, \mathcal{I}}(x), 0, \cdot, \cdot\right]$ is then a straightforward computation through (S4), (S5), and the results can be compared to the closed-form solutions from [8,22]: $\phi_{k}=\phi_{L-k+1}$, an integer $j$ co-prime to $L$, where $\phi_{1}=0$ and

$$
\phi_{k+1}=\phi_{k}+2 \tan ^{-1}\left[\tan \left(\frac{2 j k \pi}{L}\right) \sqrt{1-\beta_{L, \mathcal{I}}^{-2}}\right] .
$$

The phases $\vec{\psi}$ for the narrow-band variant $\left[\cdot, \cdot, \mathrm{DC}_{L, \mathcal{I}}(x), 0\right]$ are obtained by a simple "toggling" transformation [13] $\psi_{k}=-(-1)^{k} \phi_{k}-2 \sum_{h=1}^{k-1}(-1)^{h} \phi_{h}$.

\section{B. Broadband compensated NOT gates}

Broadband compensated NOT gates maximize the bandwidth $\mathcal{B}$ over which the fidelity response function with respect to the target gate $\hat{R}_{0}(\pi)$ is close to 1 . One option consistent with this goal is the choice of fidelity response functions $F_{\pi}(\theta)=1-\mathcal{O}\left((\theta-\pi)^{2 n+2}\right)$ that are maximally flat with respect to $(\theta-\pi)$. When the correction order $n$ increases, deviations from $\theta=\pi$ are exponentially suppressed, resulting in improved approximations of the target gate over wider ranges of $\theta \in \mathcal{B}$. The central difficulty of this pursuit is finding the phases $\vec{\phi}$ that maximize $n$ for any given $L$. Unlike the population inversion gates of Sec. IV A, this appears to be significantly more difficult; optimal length solutions for the $\vec{\phi}$ have only been found in closed form for small $n \leq 4$ [19].

This problem has been attacked over the course of two decades, starting with Wimperis [13] who found the $\vec{\phi}$ in closed form for $\mathrm{BB}_{1}$, an $L=5$ sequence with $n=2$. This was extended by Brown et al. [15] with SKn for arbitrary $L=\mathcal{O}\left(n^{3.09}\right)$ through a recursive construction, and then by Jones [18,51] with $\mathrm{F}_{n}$ to $L=\mathcal{O}\left(n^{1.59}\right)$ in closed form through sequence concatenation. The most recent effort [19] proved a lower bound of $L=\Omega(n)$ and conjectured that the sequence $\mathrm{BB} n$ ( $W_{n}$ in Ref. [51]) with $L=2 n+1$ is optimal through brute force up to $L=25$. Using our methodology, we can easily prove this conjecture and efficiently compute its implementation $\vec{\phi}$.

Moreover, our methodology enables a second option. Instead of optimizing for correction order, it is possible to directly minimize the worst-case infidelity $\mathcal{I}$, which is the experimental quantity of interest, over a target bandwidth
$\mathcal{B}$. We find that doing so leads to an improvement in $\mathcal{I}$ that scales exponentially with $L$ over the maximally flat case. To prove these statements, we proceed with the design outline of Sec. III E for odd $L$ :

(S1) Choose the objective function $\forall \theta \in \mathcal{B}=\pi+[-|\mathcal{B}| / 2$, $|\mathcal{B}| / 2], \hat{U}_{\mathrm{o}}(\theta)=\hat{R}_{0}(\pi)=-i \sigma_{x}$ for the $(\cdot, \cdot, C, \cdot) 3-$ partial tuple. Provided that $\mathcal{B}$ does not contain the point $\theta=0$, this is consistent with the constraints of Theorem 3. This corresponds to finding a fidelity response function $F_{\pi}(\theta)=C^{2}[\sin (\theta / 2)]$ that is close to 1 across $\mathcal{B}$.

(S2) The best fidelity response function for the maximally flat approach in prior art is obtained from the function $C$ that maximizes the correction order

$$
\begin{aligned}
& n=\max _{C}\left\{n \mid C(y)=1-\mathcal{O}\left[(1-y)^{n+1}\right]\right\}, \\
& \mathcal{I}=1-\min _{\theta \in \mathcal{B}} F_{\pi}(\theta), \quad y \equiv \sin (\theta / 2),
\end{aligned}
$$

where $\mathcal{I}$ is the worst-case infidelity over the bandwidth $\mathcal{B}$. It is easy to verify that any such $C$ satisfies $F_{\pi}(\theta)=1-\mathcal{O}\left[(\theta-\pi)^{2 n+2}\right]$. The more direct approach uses the Chebyshev optimality criterion, where the best $C$ solves the minimax optimization problem

$$
\epsilon=\min _{C} \max _{\theta \in \mathcal{B}}|C(y)-1|, \quad \mathcal{I}=1-(1-\epsilon)^{2} .
$$

(S3) Find the function $C$ that solves Eqs. (31) and (32). For consistency with Theorem 1, the optimization is over real, odd polynomials $C$ bounded by $|C(y)| \leq 1, \forall y \in[-1,1]$.

(S4) Using Lemma 3, compute the achievable tuple $(A, 0, C, D)$ from the partial specification $(\cdot, 0, C, \cdot)$. (S5) Compute $\vec{\phi}$ from $(A, 0, C, D)$ using Lemma 1.

We now present the solutions to (S3) of this procedure. This is the most difficult step, as once $C$ is provided, the implementation $\vec{\phi}$ is a straightforward calculation. Equation (31) is solved by the odd polynomial that satisfies the following $n+1$ independent linear constraints:

$$
C(1)=1,\left.\quad \frac{d^{k}}{d y^{k}} C(y)\right|_{y=1}=0, \quad k=1,2, \ldots, n .
$$

As a degree $L$ odd polynomial has $(L+1) / 2$ free parameters, a degree $L=2 n+1$ polynomial is necessary and sufficient. This is solved by the polynomial

$$
\mathrm{M}_{L, \mathrm{f}}(y)=\sum_{j=0}^{(L-1) / 2}\left(\begin{array}{c}
L \\
j
\end{array}\right)\left(\frac{1+y}{2}\right)^{L-j}\left(\frac{1-y}{2}\right)^{j},
$$

with an example $\mathrm{M}_{9, \mathrm{f}}$ plotted in Fig. 1. The index $L$ indicates the degree, and the subscript $\mathrm{f}$ indicates that this is a maximally flat polynomial. As $\mathrm{M}_{L, \mathrm{f}}(y)$ is monotonically decreasing from $y<1$, the relation between infidelity $\mathcal{I}$ 
and bandwidth $\mathcal{B}$ is obtained by solving $\mathcal{I}=1-$ $M_{L, \mathrm{f}}^{2}[\cos (|\mathcal{B}| / 4)]$ to leading order:

$$
\mathcal{I}=\left(\frac{|\mathcal{B}|}{8}\right)^{L+1} \frac{2^{L+5 / 2}}{\sqrt{\pi L}}\left[1+\mathcal{O}\left(\left(\frac{|\mathcal{B}|}{8}\right)^{2}+\frac{1}{L}\right)\right]
$$

Thus, given some target bandwidth $\mathcal{B}$ of high-fidelity operation, the composite quantum gate represented by
$\mathrm{BB} n=\left[\cdot, 0, \mathrm{M}_{2 n+1, \mathrm{f}}(y), \cdot\right]$ implements NOT with a worstcase fidelity that decreases exponentially with sequence length. This proves the $L=2 n+1$ conjecture of Ref. [19].

The odd polynomials of degree $L$ that satisfy the Chebyshev error-norm optimality criterion in Eq. (32) can also be found. We label these polynomials $\mathrm{M}_{L, \mathcal{I}}$, where $L$ indicates the degree, and $\mathcal{I}$ is the worst-case infidelity, which is directly related to the bandwidth $\mathcal{B}$. For $L=5$, we have a complicated-looking expression,

$$
\begin{aligned}
\mathrm{M}_{5, \mathcal{I}} & =\frac{\left(2 y_{1}+1\right) y^{5}-\left(4 y_{1}^{3}+3 y_{1}^{2}+2 y_{1}+1\right) y^{3}+\left(2 y_{1}^{5}+4 y_{1}^{4}+6 y_{1}^{3}+3 y_{1}^{2}\right) y}{2 y_{1}^{3}\left(y_{1}+1\right)^{2}}, \\
\mathcal{I} & =\frac{\left(y_{1}-1\right)^{3}\left(1+3 y_{1}+y_{1}^{2}\right)^{2}\left(1-2 y_{1}-4 y_{1}^{2}\right)\left(3+9 y_{1}+8 y_{1}^{2}\right)^{3}}{3125 y_{1}^{6}\left(1+y_{1}\right)^{4}\left(1+2 y_{1}\right)^{3}},
\end{aligned}
$$

parametrized implicitly through $y_{1} \in[0.3671,1]$. For larger $L$, such as $\mathrm{M}_{9,10^{-2}}$ in Fig. 1, the $\mathrm{M}_{L, \mathcal{I}}$ can always be computed numerically through the famous ParksMcClellan algorithm [40] for finite impulse-response filters. Remarkably, the Chebyshev error of this approximation problem is known [52]:

$$
\begin{aligned}
\epsilon & =\left(\frac{1}{\sqrt{2 \pi}}+o(1)\right) \frac{8 \cos ^{2}(|\mathcal{B}| / 8) \tan ^{L+1}(|\mathcal{B}| / 8)}{\sqrt{(L-1) \cos (|\mathcal{B}| / 4)}} \\
\mathcal{I} & =\left(\frac{|\mathcal{B}|}{8}\right)^{L+1} \frac{2^{7 / 2}}{\sqrt{\pi L}}\left[1+\mathcal{O}\left(\left(\frac{|\mathcal{B}|}{8}\right)^{2}+\frac{1}{L}\right)\right]
\end{aligned}
$$

By comparing Eqs. (35) and (37) in Fig. 2, it can be seen that for any target $\mathcal{B}$ and sequence length $L$, the composite quantum gate $\mathrm{OB} n=\left[\cdot, 0, \mathrm{M}_{2 n+1, \mathcal{I}}(y), \cdot\right]$ has a worst-case infidelity that improves on $\mathrm{BB} n$ by an exponential factor $\mathcal{O}\left(2^{1-L}\right)$. In contrast to the $\mathrm{BB} n$ sequences that are fixed for each $n, \mathrm{OB} n$ allows for an optimal design trade-off between bandwidth $\mathcal{B}$ and infidelity $\mathcal{I}$. As seen in Fig. 1, this occurs by introducing equiripples of equal amplitude bounded by $\mathcal{I}$, similar to the $\mathrm{DC}_{L, \mathcal{I}}$ polynomials for population inversion gates. Thus, given the same performance targets, an extremely short $\mathrm{OB} n$ gate can perform just as well as a significantly longer $\mathrm{BB} n$ gate. In other words, maximizing the correction order only improves the achieved bandwidth indirectly, leading to a poor trade-off between $\mathcal{I}$ and $\mathcal{B}$, whereas better results are naturally achieved by optimizing for polynomials that directly solve Eq. (32) by minimizing infidelity over a target bandwidth.

\section{Composite quantum gates with subwavelength spatial selectivity}

Narrow-band compensated gates maximize the bandwidth $\mathcal{B}$ over which the fidelity response function with respect to identity $\hat{\mathbb{1}}$ is close to 1 , except at a single point $\theta$ where an arbitrary target rotation $\hat{R}_{0}(\chi)$ is applied. Although the direct approach computes new polynomials $(A, \cdot, C, \cdot)$ that satisfy these properties, we can reuse the polynomials $M_{L, \mathcal{I}}$ from Sec. IV B by making certain assumptions on the physical system. In the following, we also assume that $|\chi| \leq \pi$.

Consider a Gaussian beam of fixed width $\lambda$. As a function of position $r$, this beam has a spatially varying Rabi frequency $\Omega(r)=\Omega_{0} e^{-r^{2} / 2 \lambda^{2}}$. Thus, when applied for time $t_{0}$, a primitive gate $\hat{R}_{\phi}[\theta(r)]$ that also varies as a

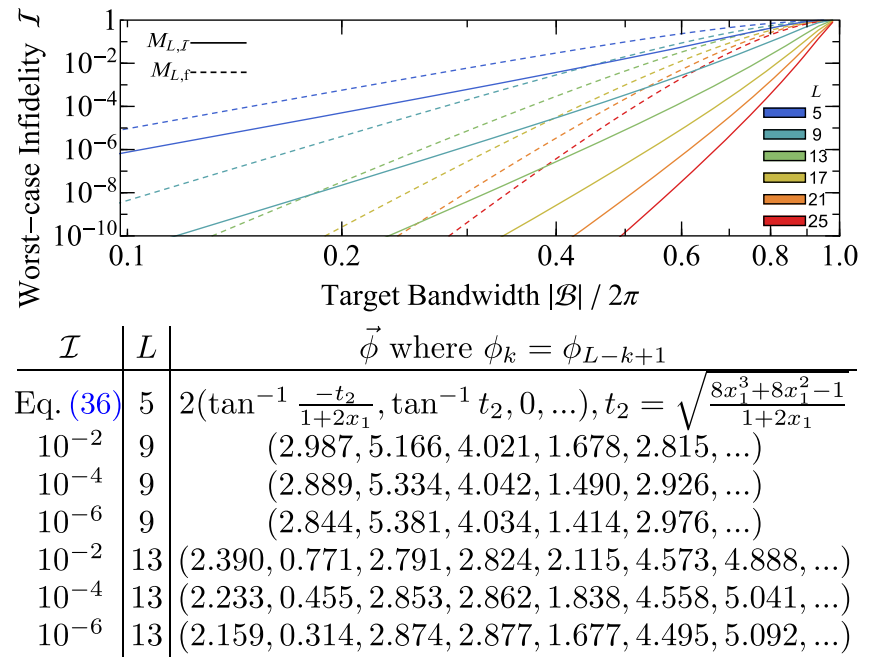

FIG. 2. Worst-case infidelity $\mathcal{I}$ of NOT gates $\mathrm{OB} n=$ $\left[\cdot, 0, M_{2 n+1, \mathcal{I}}(\sin (\theta / 2)), \cdot\right]$ [solid line; Eq. (37)] optimized for target bandwidth $\theta \in \mathcal{B}$ compared to the flatness-optimized NOT gate $\mathrm{BB} n=\left[\cdot, 0, M_{2 n+1, \mathrm{f}}(\sin (\theta / 2))\right.$ ). ] [dashed line; Eq. (35)], plotted for $L=2 n+1=5,9, \ldots, 25$ (from top to bottom). Observe that $\mathcal{I}$ for $\mathrm{OB} n$ is exponentially smaller by a factor $\approx 4^{n}$ than $\mathrm{BB} n$. Alternatively, an $\mathrm{OB} n$ gate can approximate NOT with infidelity of, at most, $\mathcal{I}$ over a much wider bandwidth than $\mathrm{BB} n$. The table provides examples of $\vec{\phi}$ for $\mathrm{OB} n$ rounded to three decimal places. 
function of position is generated, where $\theta(r)=\theta_{0} e^{-r^{2} / 2 \lambda^{2}}$ and $\theta_{0}=\Omega_{0} t_{0}$. At $r=0$, one can choose $t_{0}, \phi$ such that the target rotation $\chi=\theta_{0}$ is implemented, and because of the exponential decay of the Gaussian beam, moving away from the beam center approximates the identity gate with infidelity $\mathcal{I}(r)=\sin ^{2}(\theta(r) / 2)$. Thus, at distance $r / \lambda \geq$ $d / \lambda=\overline{\mathcal{B}}_{1}=\log ^{1 / 2}\left(\pi^{2} / 4 \mathcal{I}\right)$ from the beam center, the worst-case infidelity is $\mathcal{I}$. As the minimum possible beam width $\lambda$ is the wavelength of light, selective addressing below the diffraction limit appears impossible. However, even this can be overcome with a carefully designed composite quantum gate.

Narrow-band composite gates of length $L$ applicable to this scenario have been widely studied. For instance, Refs. [10,13] report beam-width reductions by a factor $(d / \lambda) \approx 0.7 \overline{\mathcal{B}}_{1}[10,13]$. Further reduction is possible with longer composite gates [19] but with poor scaling, $(d / \lambda)=\mathcal{O}\left(L^{-1 / 4}\right)$.

A better narrow-band composite gate results from using the broadband identity gate ID $=\left[\mathrm{M}_{L, \mathcal{I}}(x), 0, \cdot, \cdot\right]$ designed from the $\mathrm{M}_{L, \mathcal{I}}$ polynomial presented in Sec. IV B. Then, the fidelity response function with respect to identity is $F_{0}(\theta)=\mathrm{M}_{L, \mathcal{I}}^{2}(x)$, which, as we now show, corresponds to a quadratic improvement of $(d / \lambda)=\mathcal{O}\left(L^{-1 / 2}\right)$.

Let us compose ID with the Gaussian beam to produce the spatially varying quantum response function

$$
\hat{U}_{\text {space }}(r)=\operatorname{ID}\left(\theta_{0} e^{-r^{2} / 2 \lambda^{2}}\right)=\operatorname{ID}\left(\theta_{0}\right)+\mathcal{O}\left(r^{2}\right),
$$

for some choice $\left|\theta_{0}\right| \leq \pi$. Note that $\hat{U}_{\text {space }}(r)$ is stable with respect to beam-pointing errors in $r$ because of the vanishing first derivative. The degree of spatial selectivity is computed from the bandwidth in Eq. (37) by substituting $|\mathcal{B}|=2 \theta_{0} e^{-\overline{\mathcal{B}}_{\text {space }}^{2} / 2 \lambda^{2}}$ and solving for $r / \lambda$. Thus, identity is implemented with infidelity of, at most, $\mathcal{I}$ at all $r \geq d \geq$ $\lambda \overline{\mathcal{B}}_{\text {space, }}$, as seen in Fig. 3, where to leading order $\mathcal{O}\left(L^{-1 / 2}\right)$,

$$
\frac{\overline{\mathcal{B}}_{\text {space }}}{\lambda}=2 \sqrt{\frac{\log (1 / \mathcal{I})+\frac{1}{2} \log \left[2^{7} /(L \pi)\right]}{L+1}-\ln \frac{4}{\theta_{0}}} .
$$

Meanwhile at $r=0$, we obtain the gate

$$
\hat{U}_{\text {space }}(0)=R_{\gamma}\left(2 \cos ^{-1}\left\{\mathrm{M}_{L, \mathcal{I}}\left[\cos \left(\theta_{0} / 2\right)\right]\right\}\right) \text {, }
$$

where $\gamma=\arg \left[C\left(\sin \left(\theta_{0} / 2\right)\right)+i D\left(\sin \left(\theta_{0} / 2\right)\right)\right]$. The desired rotation $R_{0}(\chi)$ is thus obtained by choosing $\theta_{0}$ such that $\cos (\chi / 2)=\mathrm{M}_{L, \mathcal{I}}\left[\cos \left(\theta_{0} / 2\right)\right]$ and rotating all phases $\phi_{k} \leftarrow \phi_{k}+\gamma$, which follows from $e^{-i(\gamma / 2) \sigma_{z}} \hat{U}_{\text {space }}(0) \times$ $e^{i(\gamma / 2) \sigma_{z}}=R_{0}(\chi)$.

The optimality of these results follows from the construction of $\mathrm{M}_{L, \mathcal{I}}$ as optimal bandwidth polynomials. In particular, using the flat polynomial $\mathrm{M}_{L, \mathrm{f}}(x)$ leads

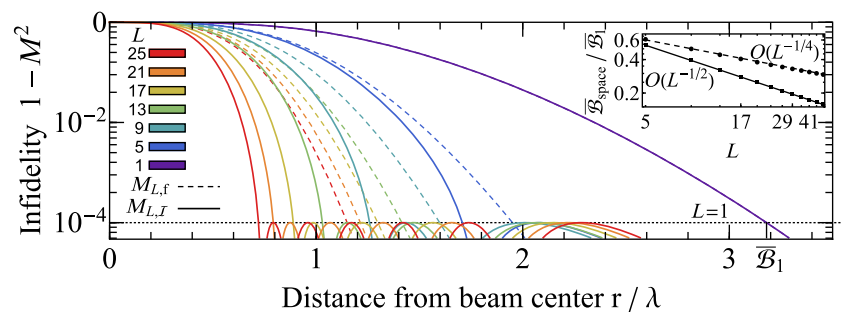

\begin{tabular}{c|c|c}
$\mathcal{I}$ & $L$ & $\vec{\phi}$ where $\phi_{k}=\phi_{L-k+1}$ \\
\hline Eq. (36) & 5 & $2\left(0, \tan ^{-1} t_{1}, \tan ^{-1} \frac{t_{1}}{1-4 x_{1}^{2}}, \ldots\right), t_{1}=\sqrt{8 x_{1}^{3}+8 x_{1}^{2}-1}$ \\
$10^{-2}$ & 9 & $(0,0.772,4.357,2.827,3.886, \ldots)$ \\
$10^{-4}$ & 9 & $(0,1.087,4.501,2.707,3.961, \ldots)$ \\
$10^{-6}$ & 9 & $(0,1.235,4.601,2.695,4.029, \ldots)$ \\
$10^{-2}$ & 13 & $(0,1.450,3.683,2.501,3.220,5.577,5.728, \ldots)$ \\
$10^{-4}$ & 13 & $(0,1.872,4.326,2.844,3.602,6.271,0.257, \ldots)$ \\
$10^{-6}$ & 13 & $(0,2.077,4.616,2.978,3.742,0.250,0.578, \ldots)$
\end{tabular}

FIG. 3. Infidelity of spatially selective composite gates $\left(M_{L, 10^{-4}}\left\{\cos \left[\left(\theta_{0} / 2\right) e^{-r^{2} / 2 \lambda^{2}}\right]\right\}, 0, \cdot, \cdot\right)$ plotted for $\theta_{0}=\pi$ and $L=$ $1, \ldots, 25$ (solid lines, from right to left). The effective beam width $\overline{\mathcal{B}}_{\text {space }}=\mathcal{O}\left(L^{-1 / 2}\right)$ (inset) beyond which the identity gate is well approximated is dramatically reduced over that of a single gate $\overline{\mathcal{B}}_{1}$. By varying $\theta_{0}$, arbitrary unitary gates can be applied at $r=0$ with high beam-pointing stability. Poorer scaling $\overline{\mathcal{B}}_{\text {space }}=$ $\mathcal{O}\left(L^{-1 / 4}\right)$ results from using the flat $\left(M_{L, \mathrm{f}}, 0, \cdot, \cdot\right)$ (dashed lines). The table provides examples of $\vec{\phi}$ to three decimal places.

to the scaling $\overline{\mathcal{B}}_{\text {space }}=\mathcal{O}\left(L^{-1 / 4}\right)$ found in prior art and Fig. 3 (inset).

\section{CONCLUSION}

We have presented and applied a methodology, analogous to the Shinnar-Le Roux algorithm but with different controls, for the systematic design of resonant equiangular composite quantum gates of length $L$ on a single spin. In particular, we show that all steps are efficient with time complexity $\mathcal{O}[\operatorname{poly}(L)]$ and provide an extremely rigorous characterization of achievable quantum response functions. Moreover, the elegant and practical connection made with discrete-time signal processing allows us to inherit and adapt many existing algorithms and polynomials used in the design of classical response functions for this quantum problem. Much potential remains untapped there, and interdisciplinary exchange could spur the discovery of further connections, leading to the development of previously intractable applications. Indeed, this relationship has already proven fruitful in surprising directions, such as recent work furnishing optimal algorithms for important problems such as Hamiltonian simulation [53,54] on a quantum computer.

In fact, our work bridges discrete-time signal processing and quantum query algorithms for evaluating symmetric Boolean functions. The idea here is that the SU(2) space of a single qubit, as we study here, is isomorphic to the $\mathrm{SU}(2)$ subspace spanned by a uniform superposition of marked 
states and a uniform superposition of unmarked state in such a query problem. Query algorithms can be built to calculate a Boolean function $f:\{0,1\}^{n} \rightarrow\{0,1\}$ that depends only on the number of marked states [i.e., $f(x)=$ $\tilde{f}(|x|)$ for some $\tilde{f}:\{0,1, \ldots, n\} \rightarrow\{0,1\}]$, and we do so with a Grover-type algorithm of partial reflections (e.g., Refs. [21,55-57]). Thus, the same methods introduced here also give a way to determine how many reflections (analogous to our $L$ ) and what reflections (analogous to our $\phi_{j}$ ) are required to compute any particular symmetric Boolean function, achieving the known lower bounds for this problem, which (not) coincidentally are also derived using polynomials [58]. As examples of this correspondence, $\mathrm{DC}_{L, \mathcal{I}}$ is an optimal solution for the OR function [22]; $\mathrm{M}_{L, \mathcal{I}}$ is optimal for the majority function.

Various thought-provoking extensions are also motivated. The set of achievable quantum response functions is changed by introducing elements such as additional (possibly continuous) control parameters, disturbances, coupled spins [10,59,60], or open systems [20,61]. These all enable their own unique applications, but they also somehow appear difficult to solve systematically and intuitively. Our success in the case of composite gates contributes supporting evidence that a useful characterization, as well as efficient methods for these more complex design problems, could exist.

\section{ACKNOWLEDGMENTS}

G. H. L. acknowledges funding by NSF RQCC Project No. 1111337. T. J. Y. acknowledges funding by the National Defense Science and Engineering Graduate fellowship. This work was sponsored in part by the National Reconnaissance Office. We thank Alan Oppenheim and Tom Baran for inspiring discussions, as well as connections made possible by their 6.341x open online MITx course. We thank Yuan Su for comments on the manuscript.

[1] L. M. K. Vandersypen and I. L. Chuang, NMR Techniques for Quantum Control and Computation, Rev. Mod. Phys. 76, 1037 (2005).

[2] R. Freeman, Spin Choreography (Oxford University Press, Oxford, England, 1998).

[3] M. A. Nielsen and I. L. Chuang, Quantum Computation and Quantum Information, 1st ed. (Cambridge University Press, Cambridge, England, 2004).

[4] M. H. Levitt, Composite Pulses, in eMagRes (John Wiley \& Sons, New York, 2007).

[5] N. Khaneja, T. Reiss, C. Kehlet, T. Schulte-Herbrüggen, and S. J. Glaser, Optimal Control of Coupled Spin Dynamics: Design of NMR Pulse Sequences by Gradient Ascent Algorithms, J. Magn. Reson. 172, 296 (2005).

[6] K. Arai, C. Belthangady, H. Zhang, N. Bar-Gill, S. J. DeVience, P. Cappellaro, A. Yacoby, and R. L. Walsworth, Fourier Magnetic Imaging with Nanoscale Resolution and
Compressed Sensing Speed-up Using Electronic Spins in Diamond, Nat. Nanotechnol. 10, 859 (2015).

[7] T. Häberle, D. Schmid-Lorch, K. Karrai, F. Reinhard, and J. Wrachtrup, High-Dynamic-Range Imaging of Nanoscale Magnetic Fields Using Optimal Control of a Single Qubit, Phys. Rev. Lett. 111, 170801 (2013).

[8] G. H. Low, T. J. Yoder, and I. L. Chuang, Quantum Imaging by Coherent Enhancement, Phys. Rev. Lett. 114, 100801 (2015).

[9] B. T. Torosov and N. V. Vitanov, Smooth Composite Pulses for High-Fidelity Quantum Information Processing, Phys. Rev. A 83, 053420 (2011).

[10] J. T. Merrill, S. C. Doret, G. Vittorini, J. P. Addison, and K. R. Brown, Transformed Composite Sequences for Improved Qubit Addressing, Phys. Rev. A 90, 040301 (2014).

[11] J. Vanier, Atomic Clocks Based on Coherent Population Trapping: A Review, Appl. Phys. B 81, 421 (2005).

[12] S. Kimmel, G. H. Low, and T. J. Yoder, Robust Calibration of a Universal Single-Qubit Gate Set via Robust Phase Estimation, Phys. Rev. A 92, 062315 (2015).

[13] S. Wimperis, Broadband, Narrowband, and Passband Composite Pulses for Use in Advanced NMR Experiments, J. Magn. Reson., Ser. A 109, 221 (1994).

[14] H. K. Cummins, G. Llewellyn, and J. A. Jones, Tackling Systematic Errors in Quantum Logic Gates with Composite Rotations, Phys. Rev. A 67, 042308 (2003).

[15] K. R. Brown, A. W. Harrow, and I. L. Chuang, Arbitrarily Accurate Composite Pulse Sequences, Phys. Rev. A 70, 052318 (2004).

[16] G. S. Uhrig, Keeping a Quantum Bit Alive by Optimized

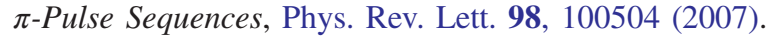

[17] X. Wang, L. S. Bishop, E. Barnes, J. P. Kestner, and S. DasSarma, Robust Quantum Gates for Singlet-Triplet Spin Qubits Using Composite Pulses, Phys. Rev. A 89, 022310 (2014).

[18] J. A. Jones, Nested Composite NOT Gates for Quantum Computation, Phys. Lett. A 377, 2860 (2013).

[19] G. H. Low, T. J. Yoder, and I. L. Chuang, Optimal Arbitrarily Accurate Composite Pulse Sequences, Phys. Rev. A 89, 022341 (2014).

[20] A. Soare, H. Ball, D. Hayes, J. Sastrawan, M. C. Jarratt, J. J. McLoughlin, X. Zhen, T. J. Green, and M. J. Biercuk, Experimental Noise Filtering by Quantum Control, Nat. Phys. 10, 825 (2014).

[21] G. Brassard, P. Høyer, and A. Tapp, Quantum Counting, in Automata, Languages and Programming (Springer, Berlin, 1998), pp. 820-831.

[22] T. J. Yoder, G. H. Low, and I. L. Chuang, Fixed-Point Quantum Search with an Optimal Number of Queries, Phys. Rev. Lett. 113, 210501 (2014).

[23] S. J. Glaser, U. Boscain, T. Calarco, C. P. Koch, W. Köckenberger, R. Kosloff, I. Kuprov, B. Luy, S. Schirmer, T. Schulte-Herbrüggen et al., Training Schrödinger's Cat: Quantum Optimal Control, Eur. Phys. J. D 69, 279 (2015).

[24] J. Ruths and J. S. Li, Optimal Control of Inhomogeneous Ensembles, IEEE Trans. Autom. Control 57, 2021 (2012).

[25] I. M. Ross and M. Karpenko, A Review of Pseudospectral Optimal Control: From Theory to Flight, Annu. Rev. Control 36, 182 (2012). 
[26] A. Oppenheim and R. Schafer, Discrete-Time Signal Processing, 3rd ed. Prentice-Hall Signal Processing Series (Prentice-Hall, Englewood Cliffs, NJ, 2010).

[27] F. Harris, On the Use of Windows for Harmonic Analysis with the Discrete Fourier Transform, Proc. IEEE 66, 51 (1978).

[28] C. Kabytayev, T. J. Green, K. Khodjasteh, M. J. Biercuk, L. Viola, and K. R. Brown, Robustness of Composite Pulses to Time-Dependent Control Noise, Phys. Rev. A 90, 012316 (2014).

[29] J.S. Li and N. Khaneja, Ensemble Control of Bloch Equations, IEEE Trans. Autom. Control 54, 528 (2009).

[30] J. S. Li, J. Ruths, T. Y. Yu, H. Arthanari, and G. Wagner, Optimal Pulse Design in Quantum Control: A Unified Computational Method, Proc. Natl. Acad. Sci. U.S.A. 108, 1879 (2011).

[31] M. Shinnar, S. Eleff, H. Subramanian, and J. S. Leigh, The Synthesis of Pulse Sequences Yielding Arbitrary Magnetization Vectors, Magn. Reson. Med. 12, 74 (1989).

[32] J. Pauly, P. L. Roux, D. Nishimura, and A. Macovski, Parameter Relations for the Shinnar-Le Roux Selective Excitation Pulse Design Algorithm [NMR Imaging], IEEE Trans. Med. Imaging 10, 53 (1991).

[33] V. N. Ikonomidou and G. D. Sergiadis, Improved ShinnarLe Roux Algorithm, J. Magn. Reson. 143, 30 (2000).

[34] K. J. Lee, General Parameter Relations for the Shinnar-Le Roux Pulse Design Algorithm, J. Magn. Reson. 186, 252 (2007).

[35] W. A. Grissom, Z. Cao, and M. D. Does, $\left|B_{1}^{+}\right|$-Selective Excitation Pulse Design Using the Shinnar-Le Roux Algorithm, J. Magn. Reson. 242, 189 (2014).

[36] M. H. Stone, The Generalized Weierstrass Approximation Theorem, Math. Mag. 21, 167 (1948).

[37] M. Marshall, Positive Polynomials and Sums of Squares (American Mathematical Society, Providence, 2008), p. 146.

[38] C. Neff and J.H. Reif, An Efficient Algorithm for the Complex Roots Problem, J. Complexity 12, 81 (1996).

[39] G. Meinardus, Approximation of Functions: Theory and Numerical Methods (Springer, Berlin, 1967), Vol. 13.

[40] J. McClellan, T. Parks, and L. Rabiner, A Computer Program for Designing Optimum FIR Linear Phase Digital Filters, IEEE Trans. Audio Electroacoust. 21, 506 (1973).

[41] L. J. Karam and J. H. McClellan, Chebyshev Digital FIR Filter Design, Signal Process. 76, 17 (1999).

[42] F. Grenez, Design of Linear or Minimum-Phase FIR Filters by Constrained Chebyshev Approximation, Signal Process. 5, 325 (1983).

[43] M. Lang, Ph.D. thesis, Vienna University of Technology (1999).

[44] E. Hofstetter, A. V. Oppenheim, and J. Siegel, A New Technique for the Design of Nonrecursive Digital Filters, in Proceedings of the 5th Annual Princeton Conference on Information Sciences and Systems (IEEE, New Jersey, 1972), p. 187.

[45] W. Fraser, A Survey of Methods of Computing Minimax and Near-Minimax Polynomial Approximations for Functions of a Single Independent Variable, J. ACM 12, 295 (1965).

[46] Y. C. Lim, J. H. Lee, C. K. Chen, and R. H. Yang, A Weighted Least Squares Algorithm for Quasi-Equiripple FIR and IIR Digital Filter Design, IEEE Trans. Signal Process. 40, 551 (1992).

[47] P. Vaidyanathan and T. Nguyen, Eigenfilters: A New Approach to Least-Squares FIR Filter Design and Applications Including Nyquist Filters, IEEE Trans. Comput.-Aided Des. Integr. Circuits Syst. 34, 11 (1987).

[48] N. V. Vitanov, Arbitrarily Accurate Narrowband Composite Pulse Sequences, Phys. Rev. A 84, 065404 (2011).

[49] C. L. Dolph, A Current Distribution for Broadside Arrays Which Optimizes the Relationship Between Beam Width and Side-Lobe Level, Proc. IRE 34, 335 (1946).

[50] P. Lynch, The Dolph-Chebyshev Window: A Simple Optimal Filter, Mon. Weather Rev. 125, 655 (1997).

[51] S. Husain, M. Kawamura, and J. A. Jones, Further Analysis of Some Symmetric and Antisymmetric Composite Pulses for Tackling Pulse Strength Errors, J. Magn. Reson. 230, 145 (2013).

[52] A. Eremenko and P. Yuditskii, Polynomials of the best uniform approximation to $\operatorname{sgn}(x)$ on two intervals, J. Anal. Math. 101, 313 (2007).

[53] G. H. Low and I. L. Chuang, Optimal Hamiltonian Simulation by Quantum Signal Processing, arXiv:1606.02685 [Phys. Rev. Lett. (to be published)].

[54] G. H. Low and I. L. Chuang, Hamiltonian Simulation by Qubitization, arXiv:1610.06546.

[55] L. K. Grover, A Fast Quantum Mechanical Algorithm for Database Search, Proceedings of the Twenty-eighth Annual ACM Symposium on Theory of Computing (Association for Computing Machinery, New York, 1996), p. 212.

[56] P. Høyer, Arbitrary Phases in Quantum Amplitude Amplification, Phys. Rev. A 62, 052304 (2000).

[57] L. K. Grover, Fixed-Point Quantum Search, Phys. Rev. Lett. 95, 150501 (2005).

[58] R. Beals, H. Buhrman, R. Cleve, M. Mosca, and R. de Wolf, Quantum Lower Bounds by Polynomials, J. ACM 48, 778 (2001).

[59] Y. Tomita, J. T. Merrill, and K. R. Brown, Multi-Qubit Compensation Sequences, New J. Phys. 12, 015002 (2010).

[60] S. S. Ivanov and N. V. Vitanov, Composite Two-Qubit Gates, Phys. Rev. A 92, 022333 (2015).

[61] K. Khodjasteh, D. A. Lidar, and L. Viola, Arbitrarily Accurate Dynamical Control in Open Quantum Systems, Phys. Rev. Lett. 104, 090501 (2010). 